\title{
Rectangular Traffic Sign Recognition
}

\author{
Roberto Ballerini ${ }^{1}$, Luigi Cinque ${ }^{2}$, Luca Lombardi ${ }^{1}$, and Roberto Marmo ${ }^{1}$ \\ 1 Dipartimento di Informatica e Sistemistica, University of Pavia, 27100 Pavia, Italy \\ \{luca. lombardi, roberto.marmo\}@unipv.it \\ 2 Dipartimento di Informatica "La Sapienza", University of Roma, Italy \\ cinque@di.uniroma1.it
}

\begin{abstract}
In this research the problem of the automatic detection and classification of rectangular road sign has been faced. The first step concerns the robust identification of the rectangular sign, through the search of gray level discontinuity on the image and Hough transform. Due to variety of rectangular road signs, we first recognize the guide sign and then we consider advertising the other rectangular signs. The classification is based on analysis of surface color and arrows direction of the sign. We have faced different problems, primarily: shape alterations of the sign owed to the perspective, shades, different light conditions, occlusion. The obtained results show the feasibility of the system.
\end{abstract}

\section{Introduction}

In this research the problem of the automatic detection and classification of rectangular road sign (Fig. 1) has been faced. In recent years, much papers on road sign recognition has been proposed with the aim at driving assistance system and autonomous vehicle [2/3,4]. Related to road guide sign set up for guidance at intersection, some approaches can detect a guide sign surrounded by flat intensity sky region in plane image at upper center [14]. Moreover, some approaches have been proposed concerning the analysis of the arrows representing directions and the recognition of the character representing destinations and distances, in order to understand information on the sign by assigning the characters to the arrowheads 1679. Our approach can detect sign candidates located at the right hand of the highway even in presence of more complex background. We define five classes of road signs (Fig. 1): advertising, extra-urban, urban, tourist locality, highway. The elements of the last set can strongly differ between them and the others, instead the components of the first four partitions are similar. In such representation, the five classes appear as separated partitions, underlining the difficulty to directly delineate boundaries between them. The Italian Highway Code [5] give information about: 1) shape: rectangular signs are used only with aspect ratio in $[0.5,2.0]$ and the surface is bounded in $6 \mathrm{~m}^{2} ; 2$ ) color: colors choice for guide sign: blue for extra-urban, white for urban, brown for tourist locality, green for highway; there are not constraints related to colors for advertising sign, however the Code prohibits in explicit way to use them in combinations that objectively give advertising sign similar to the indication road 


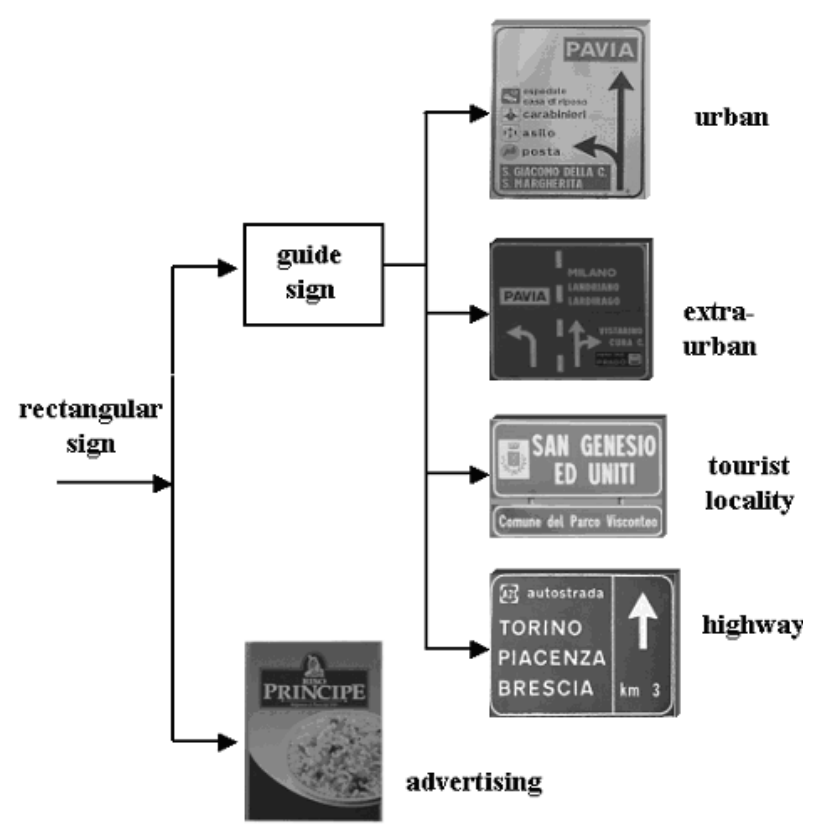

Fig. 1. Classes of rectangular road signs

sign; 3) location: they must be sets to at least height of $1.5 \mathrm{~m}$. from the road level and displaced to at least 3 meters from the right limit of the road.

\section{Acquisition System}

Our vision system is based on a single camera and bitmap color images of 640x480 pixels, 150 dpi, 24 bits for each pixel. It is important to delimit a small area of interest, minimizing the possibility to get noise from undesired objects. Due to location of the sign, we consider as region of interest the area of $320 \times 480$ pixels corresponding to the right hand of the image. Additionally, due to the frequent absence of the road borderlines it is impossible to use the borderlines for delimiting a specific area of interest on the right hand. So, it is necessary to install the camera with an opportune tilt angle q; the value is set to 30 considering the possibility to recognition signs partially hidden by obstacles and the necessity to do not deform the detected shape. Due to horizontal skew between the vision system and the road perpendicular to the sign, the sign appears on a rotated plan (Fig. 2).

The width $w^{\prime}$ of the sign is less than real $w: w^{\prime}=w^{*} x \cos (\theta)$. The scale reduction is proportional to the increase of distance among the two extremities of the sign from the camera. The minimum and maximum distances between camera and $\operatorname{sign}$ are $d_{\min }=d$ and $d_{\max }=d+\Delta d$ where $\Delta d=w \times \sin \theta$. It is necessary to evaluate the apparent scale reduction of the more distant part 

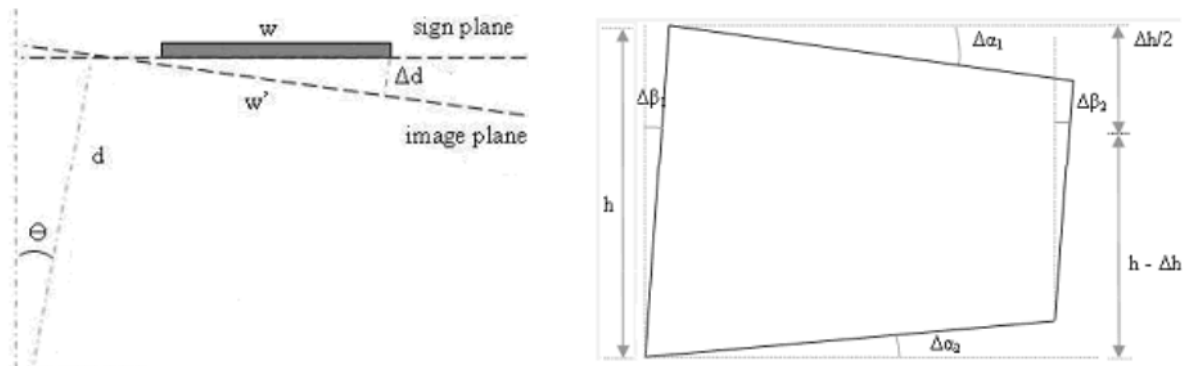

camera

Fig. 2. Left: position of the camera and rectangular sign, right: model of the sign considering rotation and perspective deformation effects

of the sign. Due to perspective alteration, the height of the sign in the left side is reduced by a factor proportional to distance increase from observer in comparison to the left side. The height variation $\Delta h$ is:

$$
\Delta h=\frac{h}{d+\Delta d} \times \Delta d
$$

To formalize the model deformation it is necessary to evaluate the angle $\Delta \alpha^{\prime}$ due to the upper side of the sign and the horizontal line (Fig. 2):

$$
\Delta \alpha^{\prime}=\arcsin \left(\frac{\sin \theta}{2} \times \frac{h}{d+w \times \sin \theta}\right)
$$

In order to evaluate the maximum value of the angular inclination of the horizontal sides, it is necessary to maximize $\Delta \alpha^{\prime}$ in the range for the parameters $d, w, h$. It is necessary to consider that the signs are not perfectly parallel to the road level, supposing that vision system is parallel to the road and therefore perpendicularly to the sign; we need to introduce Da" that include this rotation. Instead the vertical sides are only subjects to this rotation factor, so $\Delta \alpha=$ $\Delta \alpha^{\prime}+\Delta \alpha^{\prime \prime}$ and $\Delta \beta=\Delta \alpha^{\prime \prime}$ and the deformations for horizontal sides are $\Delta \alpha_{1}$ and $\Delta \alpha_{2}$, for vertical sides are $\Delta \beta_{1}$ and $\Delta \beta_{2}$.

\section{Sign Recognition}

We decompose the recognition in three steps:

1. Identification, to verify the presence of a shape interpretable as a sign;

2. Validation, a set of controls in order to verify the real nature of the object;

3. Classification, to distinguish advertising signs from others guide signs.

\subsection{Identification}

The first step concerns the robust identification of the rectangular signs. Route guide sign assumes a rectangular shape, so we extract the four sides of rectangular shape in order to consider rectangles which matches most likely to the 
sign. The intensity of such sign is uniform on its surface and it is different from background. Therefore we analyze the level of contrast and dynamically establish thresholds to identify the four sides applying Canny edge operator, searching gray level discontinuity on the image. In order to consider only the most interesting contours or rather those characterized by more elevated contrast, a threshold is used to consider only a specific percentage $p$ of pixel of the image. Based on the histogram of the module of the gradient, the first index $s$ is chosen according to value of the module that has accumulated relative frequency equal to $1-p$ so we have:

$$
\operatorname{threshold}(p)=\min _{s}:\left(\sum_{i=0}^{s} f_{r}\left(G_{m}\right) \geq 1-p\right)
$$

After the edge detection step, it is necessary to look for line segment to verify the presence of a sign. Our approach is based on Hough transform. We do not use the generalized Hough transform to directly detect the rectangular shape, because the prospective deformation of the shape creates much quantity of possible candidates and, consequently, an high computational effort. For each interesting edge pixel we vote for all the possible straight lines that pass in the edge pixel and that have compatible inclination of the sides in the formalized model of the sign (Fig. 2). In this way it is possible to achieve a precise identification even in presence of noise and in case of partially hidden signs.

At this point the identification looking for $\mathrm{n}$ horizontal lines (accepting a maximum error $\Delta \alpha$ ) and the $\mathrm{n}$ vertical lines (accepting a maximum error $\Delta \beta$ ) which get more vote. The choice of $n$ tightly depends on the complexity of the image: in case of only signs is sufficient $n=2$ (minimal theoretical value) to exactly detect it is shape; in other situations, characterized by the presence of noise objects (stakes, vehicles, buildings, etc.) good results are obtained only choosing $n>5$. If this step has not identify an useful line set, the computation conclude that there is no sign in the image. On the contrary it is necessary to establish a criterion to select only the straight lines that effectively correspond to sign edges.

Now it is necessary to intersect every couple formed by an horizontal and a vertical line to find the vertexes of the best approximating quadrilateral. The number of shapes to be examined $(\mathrm{N})$ quickly grows with n. Problem complexity is $O\left(n^{4}\right)$, so it is necessary to use the least value of $\mathrm{n}$ that allows good results. All the localized quadrilaterals are now submitted to a sequence of test, in order to identify only the quadrilaters that contain advertising sign.

Eleven tests have been developed, separate in two categories. The first series of test is constituted by nine tests that represent some sufficient conditions for quadrilaterals invalidation. The quadrilaterals are sequentially examined, and if at least a condition is verified the correspondent quadrilateral is eliminated. The second set of tests analyzes the remaining quadrilaterals, through more articulated inspections, attributing a score to each. The phase of identification ends therefore with the selection of the figure that pass all the tests and that has gotten the highest score. If no quadrilateral reaches the end we proceed to re-analyze the image with a recovery strategy. 


\subsection{Validation}

The previous process ends with the identification of the coordinates of the best fitting quadrilateral. In order to exclude the false positive, it is necessary to identify some numerical property. The false shapes in which the system frequently fall are created by buildings and vehicles on the road. A specific analysis of chromatic and intensity characteristics of such objects suggest to study an approach based on depth perception. A sign is a plane nearly perpendicular to the observer situated to a constant distance from it. On the contrary, a false positive is generated by different objects that form a sign shape without this characteristic.

Obviously, a near and well defined object have elevated gradients related of the border. The same objects locate to greater distance loss in definition and will be characterized, therefore, from minor intensity variations. The result is the uniformity of the gradients, that we can formalize as low variance. The sign image is characterized by a greater dispersion of values in the whole range.

If the sign search is failed with a specific set of parameters, we can suppose that if we repeat the sign search with a new set of parameter value, best results can be gotten. If the image has low contrast it is probably that in the previous gradient threshold calculation we do not consider all the interesting pixels. Consequently, it is necessary to repeat the line segment detection with new thresholds. In order to reduce the effect of the noise, we control the votes received by the selected straight lines. Two different method are used to determine if such lines have been produced by single object points, or if they have been produced by a group of objects:

- Backward lines checking. First method: to valuate in the Hough space the continuity of the points that give contribute to accepted lines (digitalization errors make borders of the objects frequently fragmented, making to appear a continuous line as discontinuous). Second method: to verify in any accepted line the presence of a good point concentration.

- Recovery strategy. In this way we realize a validation step of the segments detected with modified parameters. With these straight lines the survey is repeated and in case of positive result the search finishes. On contrary case this correction step is repeated, reducing at every step the parameters that control it, that are threshold on the intensity gradient module and threshold on the density of votes along the straight lines. The performances really improve, making the system more robust in situations characterized by non optimal input image.

The test set includes 1100 images: 900 containing a rectangular sign, 200 without rectangular signs. This approach can recognize the presence of a rectangular sign with $99 \%$ accuracy. The obtained results are compared with the position of the rectangular sign obtained by a human user. If the background colors are very similar to colors of advertising sign, it is not possible to detect the rectangular shape. The system is able to repeatedly detect the same sign in four consecutive images. 

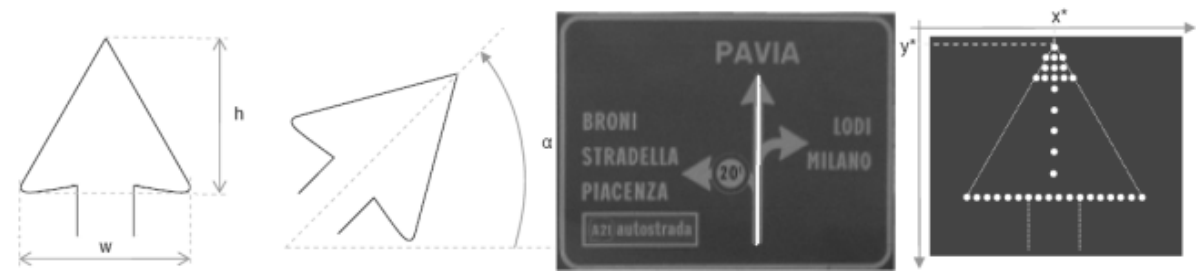

Fig. 3. Model of arrow direction (left): $\mathrm{h}$ is the height, $\mathrm{w}$ is the width, a is the direction angle. White line corresponds to the analyzed column (center) and the related analyzed rows (right) in extra-urban sign.

\subsection{Classification}

Due to variety of rectangular road signs, we first identify guide road sign and then we consider all others as advertising sign. So, it is necessary to create a set of features that characterizes the five classes (Fig. 1). Analyzing the Italian Code [5], we can understand that the first information to be examine is the percentage of the colors that are painted on the sign surface. The Code fixes the colors that must be use distinguishing them by the location and the functionality which they are created in the sign. Using the RGB color model, the frequent color is the color that covers the $50 \%$ of the sign surface (for example white for urban sign), the information color is used to show information on the sign (for example black for urban indication sign). The thresholds are empirically defined by statistic measurements on the entire set of images evaluating lighting conditions as reflections and shadows. At this point the algorithm search a characteristic element: the arrows direction. The purpose of these symbols is to facilitate the car driver in the choice of the way. To avoid confusion of the driver the Code explicitly prohibit to use the arrows direction in the advertising road sign. Moreover, the Code establishes: a specific shape, the ratio between height and width is $\mathrm{h} / \mathrm{w}=10 / 11$, arrowhead direction angle a in $[0,45,90,135,180]$ (Fig. 3).

Approaches based on contour description [7, template matching and neural networks [9], morphological operations [4] that require a considerable computational effort and they are sensitive to noise. We consider the white arrow due to highway and extra-urban sign and the black arrow due to urban sign. Our approach is based on the following steps:

1. to analyze each column of the matrix representing the image (Fig. 3);

2. if there is more than $70 \%$ of white or black pixels it is necessary to analyze the rows in the matrix composed by pixels near to the top of the white or black pixel (Fig. 3);

3. if the cluster has the shape of a triangle then there is an arrowhead.

The cluster has a triangular shape if these conditions are both satisfied:

1. $a$ correspond to number of white or black pixel in row $y, b$ correspond to number of white or black pixel in row $y+1$, so $a=b$ or $a+1=b$ in progress for each row starting from the top row $y^{*}$; 
2. the ratio between number of rows and number of columns in $[0.8,1.0]$;

3 . the ratio between height and width of the cluster of pixel is roughly 10/11.

In case of negative result, the steps are repeated using a new value of angle a, in order to rotate the candidate sign. If there is a compatible arrow the classification step finishes establishing the class of the sign. Our approach is very fast and robust: using a test set of 300 guide signs image the accuracy of correct recognition is $100 \%$. Finally, the algorithm verify the presence of typical rectangular frame that characterizes the guide sign using Hough transform to detect the four straight lines only at the boundary of the sign.

\subsection{Performance Evaluation}

The test set is formed by 900 images: 500 containing advertising, 400 containing guide sign, that is, 100 images for each guide sign class. This approach can recognize the presence of a rectangular sign with $96.6 \%$ mean accuracy, the accuracy for classification of specific guide sign is $96.5 \%$. The confusion matrix (Tab. 1) allows to summarize the classification results on the test set.

In case of erroneous classification of the guide sign, the class chosen is the advertising one, it may be seen that it does never occur that an image of an urban sign is classified as tourist or highway. Two advertising signs are misclassified as urban due to high quantity of white and black colours, one advertising sign is misclassified as tourist due to high quantity of brown and white colour. Four tourist signs are misclassified as advertising due to presence of a photo of the

Table 1. Confusion matrix: $c_{i j}$ coefficient at row $i$ and column $j$ represents the percentage of sign of class $i$ identified as sign of class $J$

\begin{tabular}{|l|l|l|l|l|l|}
\hline \multirow{2}{*}{ predicted classification } & \multicolumn{5}{l}{ real classification } \\
\cline { 2 - 6 } & advertising & extraurban & urban & tourist & highway \\
\hline advertising & 97 & 0 & 2 & 1 & 0 \\
hline extraurban & 3 & 97 & 0 & 0 & 0 \\
hline urban & 4 & 0 & 96 & 0 & 0 \\
hline tourist & 4 & 0 & 0 & 96 & 0 \\
hline highway & 3 & 0 & 0 & 0 & 97 \\
\hline
\end{tabular}
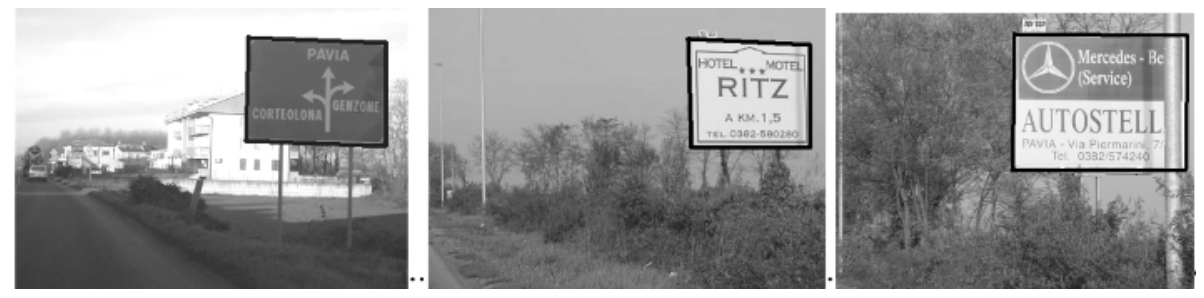

Fig. 4. Classification results indicated by the black frame: urban sign (left) and advertising (center), an advertising sign partially hidden by a pole (right). 
locality. The developed system allows good results in many situations (Fig. (4). In some images, due to presence of noise or partially hidden signs only a part of surface sign has been identified. However, this part is sufficient to complete in a corrected way the classification (Fig. 4). Some problems arise when the sign is too far, or when the sign has very low contrast considering the surrounding environment.

\section{Conclusion}

In this paper we have proposed an image analysis that allowed to recognize the rectangular road sign in colour images distinguishing them between advertising and guide sign. This technique predicts the advertising sign with $97 \%$ accuracy and the specific type of guide sign with $96.5 \%$, even in presence of perspective deformation, different conditions of brightness, partially hidden signs. A robust method for arrow direction recognition is proposed.

Experimental results show the effectiveness of the approach. This percentage is satisfactory for this preliminary application of the proposed methodology. The codes have been written in $\mathrm{C}$ language as part of a package, which can be used and extended for future applications. Future works include integration with a pre-attentive optical flow created by a camera mounted on a moving car.

\section{References}

1. Azami, S., Katahara, S., Aoki, M.: Route guidance sign identification using 2d structural description. Proceedings IEEE Int. Conf. Intelligent Transportation Systems (1996) 153-158

2. Chen, X., Yang, J., Zhang, J., Waibel, A.: Automatic detection and recognition of signs from natural scenes. IEEE Trans. Image Processing 13 (2004) 87-99

3. De La Escalera, A., Armingol, J. M., Pastor, J. M., Rodriguez, F. J.: Visual sign information extraction and identification by deformable models for intelligent vehicles. IEEE Trans. Intelligent Transportation Systems 5 (2004) 57-68

4. Franke, U., Gavrila, D., Gorzig, S., Lindner, F., Paetzold, F., Wohler, C.: Autonomous driving approaches downtown. IEEE Trans. Intelligent Transportation Systems 13 (1999) 40-48

5. Italian Highway Code. http://www.dirittoegiustiziaonline.it/infortunistica/codst2.htm

6. Kato, T., Kobayasi, A., Hase, H., Yoneda, M.: An experimental consideration for road guide sign understanding. Proc. IEEE Int. Conf. Intelligent Transportation Systems (2002) 268-273

7. Lee, J., Jo, K.: Traffic Sign Recognition by division of Characters and Symbols Regions. Proc. 7th Korea-Russia International Symposium (2003) 324-328

8. Miura, J., Kanda, T., Shirai, Y.: An active vision system for real-time traffic sign recognition. Proc. IEEE Int. Conf. Intelligent Transportation Systems (2000) 52-57

9. Priese, L., Lakmann, R., Rehrmann, V.: Ideogram Identification in a Realtime Traffic Sign Recognition System. Proc. IEEE Int. Conf. Intelligent Transportation Systems (1995) 310-314

10. Salgian, G., Ballard, D. H.: Visual routines for autonomous driving. Proc. 6th Int. Conf. Computer Vision (1998) 876-882 